\title{
Pretreatment neutrophil-to-lymphocyte ratio as an important prognostic marker in stage III locally advanced non-small cell lung cancer: confirmatory results from the PROCLAIM phase III clinical trial
}

\author{
Tithi Biswas ${ }^{1}$, Rohin Gawdi ${ }^{2}$, Charulata Jindal ${ }^{3}$, Sharanya Iyer ${ }^{1}$, Kylie H. Kang ${ }^{4}$, David Bajor ${ }^{5}$, \\ Mitchell Machtay ${ }^{6}$, Yuk Ming Choi ${ }^{7}$, Jimmy T. Efird ${ }^{8}$
}

${ }^{1}$ Department of Radiation Oncology, University Hospitals, Case Western Reserve University, Cleveland, OH, USA; ${ }^{2}$ Wake Forest School of Medicine, Winston-Salem, NC, USA; ${ }^{3}$ Harvard Medical School, Harvard University, Boston, MA, USA; ${ }^{4}$ Department of Radiation Oncology, Washington University School of Medicine and Alvin J. Siteman Comprehensive Cancer Center, St. Louis, MO, USA; ${ }^{5}$ Medical Oncology, Seidman Cancer Center, Case Western Reserve University, Cleveland, OH, USA; ' Department of Radiation Oncology, Penn State University, Hershey, PA, USA; ${ }^{7}$ Signify Health, Dallas, TX, USA; ${ }^{8}$ Cooperative Studies Program Epidemiology Center, Health Services Research and Development, DVAHCS, Durham, NC, USA

Contributions: (I) Conception and design: T Biswas, JT Efird; (II) Administrative support: C Jindal; (III) Provision of study materials or patients: T Biswas, D Bajor, M Machtay, S Iyer; (IV) Collection and assembly of data: JT Efird, C Jindal, YM Choi; (V) Data analysis and interpretation: JT Efird, C Jindal, YM Choi; (VI) Manuscript writing: All authors; (VII) Final approval of manuscript: All authors.

Correspondence to: Dr. Jimmy T. Efird. Cooperative Studies Program Epidemiology Center, Health Services Research and Development, DVAHCS, Durham, NC 27705, USA. Email: jimmy.efird@stanfordalumni.org.

Background: Neutrophil-to-lymphocyte ratio (NLR) is an important pretreatment marker of systemic inflammation and tumor aggressiveness. Increased levels of this ratio have been associated with reduced survival in several observational studies of lung cancer. However, supporting analyses from large clinical trial data are lacking.

Methods: To validate the prognostic role of NLR, the current study evaluated data from a randomized phase III study (PROCLAIM; clinicaltrial.gov ID: NCT00686959) of patients with stage IIIA/B, unresectable, non-squamous, non-small cell lung cancer (NSCLC), originally comparing combination pemetrexedcisplatin chemoradiotherapy with etoposide-cisplatin chemoradiotherapy. Adjusted hazard ratios (aHR) and 95\% confidence intervals (CI) for survival were estimated using a Cox proportional hazards model. Models were adjusted for age, race, sex, stage, treatment, and body mass index (BMI). Patients were followed for a median of 24 months.

Results: Increased NLR levels at baseline were associated with reduced overall $\left(P_{\text {Trend }}<0.0001\right)$ and progression-free survival $\left(P_{\text {Trend }}<0.005\right)$. A similar but decreasing linear trend was not observed for lymphocytes count alone.

Conclusions: These findings provide substantiating evidence that NLR, which is routinely available from standard blood testing of patients diagnosed with NSCLC, is an important inflammation-based prognostic marker for survival among patients with locally advanced disease undergoing chemoradiation. Future research will benefit by assessing the prognostic potential of NLR in the context of genetic mutations and molecular markers.

Keywords: Lung cancer; lymphopenia; neutrophilia; neutrophil-to-lymphocyte ratio (NLR); radiation

Submitted Jun 19, 2021. Accepted for publication Sep 17, 2021.

doi: $10.21037 /$ jtd-21-1018

View this article at: https://dx.doi.org/10.21037/jtd-21-1018 


\section{Introduction}

Lung cancer is the leading cause of cancer-related mortality in the United States (1). Non-small cell lung cancer (NSCLC) is the most common variant, accounting for approximately $85 \%$ of cases. One third of patients newly diagnosed with NSCLC present with locally advanced, nonmetastatic malignancy $(2,3)$. Recent treatment advances include the use of novel immune checkpoint inhibitors (ICI) (4). Nonetheless, the prognosis of patients diagnosed with stage IIIA and IIIB NSCLC remains poor, with fiveyear survival rates of $14 \%$ and $5 \%$, respectively (5). Locally advanced NSCLC is a challenging disease with a paucity of information to guide treatment decisions. A need remains to independently validate non-invasive prognostic markers for this disease, especially in the context of a sufficiently powered randomized clinical trial (6).

In 1836, inflammation was first associated with cancer by Rudolf Virchow, when his microscopic analysis of tumors showed gross infiltration of lymphocytes and macrophages (7). In recent years, the importance of inflammation in carcinogenesis and tumorigenesis has become increasingly established. The presence of chronic inflammatory states in the tumor microenvironment has been implicated in every step of carcinogenesis by promoting oncogenic mutations, accelerating neoplastic proliferation, and encouraging seeding and metastasis (8). Localization of inflammatory activity directly exposes native tissues and cells to reactive oxygen species (ROS), which activates oncogenes and introduces deleterious mutations to essential tumor suppressor genes (9). Having undergone malignant transformation, these cells stimulate localized inflammation by the secretion and attraction of pro-inflammatory factors IL-1, IL-6, IL-8, and TNFalpha, which induce further mutation and oncogenesis (10). Specific to NSCLC, loss-of-function mutations to the PTEN tumor suppressor gene results in upregulated HIF-1 and HIF-1-dependent transcription of chemokine receptor gene CXCR4, which plays a critical role in malignant cell survival and proliferation within the tumor microenvironment (11).

Changes in neutrophil and/or lymphocyte counts in peripheral blood are important surrogate markers of systemic inflammation and cancer-targeted immune reaction. Polymorphonuclear neutrophils (PMN) are leukocytes that are produced in response to systemic inflammation and facilitates oncogenesis by directly and indirectly inducing tumor proliferation and metastasis. PMN are activated in response to hematopoietic cytokine overactivation, which indicate hyper-inflammatory states that contribute to tumor angiogenesis and metastasis seen in more aggressive cancers (12). Once activated, PMN are attracted to the tumor microenvironment by IL-8 and IL-17, both of which are produced in abundance owing to K-RAS and other oncogenic mutations on malignant cells (13). Once in the tumor microenvironment, PMN further induce prooncogenic mutations through over-production of ROS and promote vessel proliferation involving various angiogenic factors. PMN also promote detachment and intravasation of malignant cells into vasculature through endothelialmesenchymal transition (14). By contrast, increased lymphocyte counts have been implicated in suppression of tumor growth and activation of pro-apoptotic activity within the tumor with CD8+ T-lymphocytes (CTL) directly induce apoptosis of tumor cells; depressed lymphocyte counts in NSCLC patients has been directly associated with decreased survival. PMN and CTL populations are directly impacted by systemic inflammatory states. IL-1, a leukocyte activation factor associated with inflammation, directly contributes to higher circulating PMN counts (neutrophilia), attracts activated PMN to regions of localized inflammation, and reduces lymphocyte counts (lymphopenia) (15-18).

Inflammation within the premalignant microenvironment also incites genetic mutations underlying tumor growth through intrinsic and extrinsic pathways $(19,20)$. In prior analyses, systemic inflammatory markers have demonstrated both prognostic and predictive value in cancer patients. Neutrophil-to-lymphocyte ratio (NLR) is a method of quantifying inflammatory and immune status. NLR has been associated with survival outcomes for several types of solid tumors (21-26). Higher NLR levels have been linked with more aggressive cancer, decreased efficacy of antineoplastic therapy and poorer clinical outcomes $(27,28)$. Although NLR has been well-studied previously, in both NSCLC as well as other malignancies, this marker remains to be independently validated in the context of a large clinical trial with systematic data collection (29).

The objective of the current analysis was to test the hypothesis that increases in NLR are associated with decreased overall survival (OS) and progression-free survival (PFS) in locally advanced NSCLC using a prospective randomized study dataset.

We present the following article in accordance with the REMARK reporting checklist (available at https://dx.doi. org/10.21037/jtd-21-1018). 


\section{Methods}

\section{Study design}

Data for the current analysis was obtained from the PROCLAIM trial (Eli Lilly), a 5-year randomized study comparing cisplatin-pemetrexed (PEM) and cisplatinetoposide (ETO) with concurrent radiation in patients with stage IIIA/B, unresectable, non-squamous, NSCLC (30). Participants were deemed eligible if they were $\geq 18$ years old and had an Eastern Cooperative Oncology Group (ECOG) Performance Status (PS) of 0/1. Evaluable disease was assessed by computed tomography (CT) imaging and with response evaluation criteria in solid tumors (RECIST) (31). Radiation doses targeted to the thoracic region ranged from 60 to 66 Gy (2 Gy/fraction daily, 5 days per week) and were delivered concurrently with chemotherapy starting with day 1 of infusion. The data used in our analysis included pre-treatment blood counts for neutrophils, platelets, and lymphocytes, reported as International System of Units (SI).

Among the 598 randomly assigned patients in PROCLAIM, approximately $2 \%$ were excluded from the analysis $(n=7$ had incomplete staging information, demographic details (race, sex), or RT start or key laboratory dates, or lacked follow-up times; $\mathrm{n}=5$ did meet entry criteria and/or physician decision). Therefore, 586 were included in the current study.

The PROCLAIM clinical trial followed the International Council for Harmonisation of Technical Requirements for Pharmaceuticals for Human Use (ICH) Good Clinical Practice (GCP) guidelines in effect at the time of the study (32). These guidelines set ethical and scientific quality standards for conducting, designing, recording, and reporting clinical trials. Additionally, our study adhered to the Reporting Recommendations for Tumor Marker Studies (REMARK) (6). Per protocol, the PROCLAIM study was designed as a superiority trial with $80 \%$ power to detect stated effects at the $\alpha=0.05$ level of statistical significance; post-hoc analyses and power were not specified in the PROCLAIM study.

The study was conducted in accordance with the Declaration of Helsinki (as revised in 2013). This was an analysis of de-identified data from the PROCLAIM study (Eli Lilly). No patients were involved or contacted for the current manuscript and the analysis was considered IRB exempt by Code of Federal Regulations 45 part 46.101(b).

\section{Statistical analysis}

Frequencies and percentages were used to summarize categorical variables, while continuous variables were reported as medians and interquartile ranges (IQR). NLR values were logarithmically transformed (base $e$ ) before performing statistical analysis to minimize heteroscedasticity and skewness of the underlying distribution.

Adjusted hazard ratios (aHRs) and 95\% confidence intervals (CI) for survival were estimated using a Cox (proportional hazards) regression model. HRs denote the relative instantaneous incidence of mortality between comparisons groups and are used to estimate relative risk. In a Cox regression analysis, the dependent variable represents the logarithm of the incidence rate, given varying lengths of time for follow-up. Independent variables are used to gauge the risk (or hazard) of experiencing an event at a given point in time, conditional on that one has not experienced the event before that time.

Models were adjusted for age, race, sex, stage, treatment, and body mass index (BMI), with the latter being a predisposing factor for inflammation. The parallel hazards assumption was not violated in our main Cox regression models (33). Product-limit estimates and corresponding Kaplan-Meier curves were used to visualize survival times over all observations.

NLR values were determined to be approximately symmetrical after logarithmic transformation (homogeneous risk within categories) and stratified by quartiles $\left(Q_{1}-Q_{4}\right)$ for the survival analyses. Neutrophil and lymphocyte values were similarly transformed. Values presented in the text and tables were back transformed to their original nonlogarithmic values to aid clinical interpretation of the results. $\mathrm{P}$ values for linear trend across risk quartiles were computed using a likelihood ratio test.

OS was defined as the time from baseline (date of random assignment) to any cause of death. PFS was similarly defined in terms of the first date of documented progressive disease or death. OS time was censored at the date the patient was last known to be alive at the time of data freeze (end of 2015), and the date of the last progression-free disease assessment, death, or subsequent systemic anticancer therapy in the case of PFS.

Numbers were rounded using the Goldilocks (EfronWhittemore) method (34). P values $<0.05$ were defined as statistically significant. SAS statistical software (version 9.4, SAS Institute Inc., Cary, NC, USA) was used for all analyses.

\section{Results}

\section{Patient characteristics}

The median patient age of the 586 study participants 
Table 1 Overall and progression-free survival for indicated characteristics (N=586)

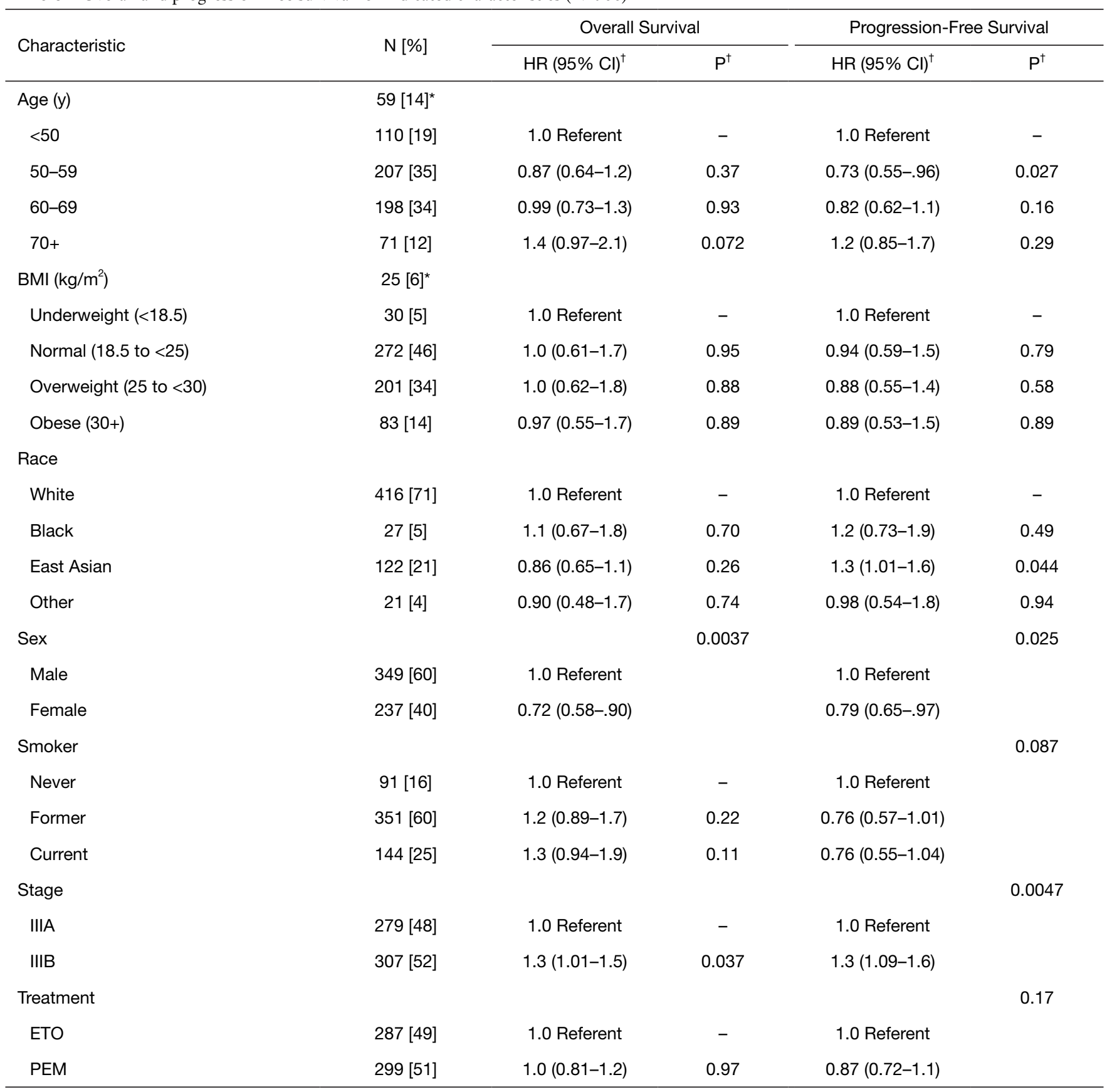

${ }^{\dagger}$, Wald test. ${ }^{*}$, data presented as Median [IQR]; BMI, body mass index; Cl, confidence interval; ETO, cisplatin + etoposide; kg, Kilogram; $\mathrm{HR}$, unadjusted hazard ratio; $\mathrm{m}$, Meter; PEM, cisplatin + pemetrexed.

was 59 years (IQR 14) (Table 1). Approximately $60 \%$ were male and slightly over $50 \%$ presented with stage IIIB disease. Adenocarcinomas were the predominant histologic type $(>75 \%)$. The main race was Caucasian (71\%). On average, patients had a BMI of 25 (IQR 6) and $60 \%$ were former smokers. There were 299 patients who received PEM (51\%), and 287 (49\%) who received ETO as part of their treatment regimens. Female patients had better overall survival outcomes than male patients (OS: HR $=0.72$; PFS: HR $=0.79)$. OS $(\mathrm{P}=0.037)$ and PFS 
Table 2 Overall and progression-free survival by inflammatory markers

\begin{tabular}{|c|c|c|c|c|c|c|c|c|}
\hline \multirow{2}{*}{ Inflammatory marker } & \multicolumn{4}{|c|}{ Overall survival } & \multicolumn{4}{|c|}{ Progression-free survival } \\
\hline & $\mathrm{HR}(95 \% \mathrm{Cl})$ & $P^{\dagger}$ & aHR (95\% Cl) & $\mathrm{P}^{\dagger}$ & HR (95\% Cl) & $\mathrm{P}^{\dagger}$ & aHR (95\% Cl) & $\mathrm{P}^{\dagger}$ \\
\hline \multicolumn{9}{|l|}{ 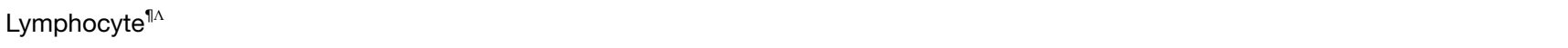 } \\
\hline Q1 (s1.5) & 1.0 Referent & - & 1.0 Referent & - & 1.0 Referent & - & 1.0 Referent & - \\
\hline Q3 (>1.9-2.4) & $0.81(0.61-1.1)$ & 0.16 & $0.77(0.58-1.03)$ & 0.081 & $0.97(0.74-1.3)$ & 0.79 & $0.92(0.69-1.2)$ & 0.53 \\
\hline Q4 (>2.4) & $0.78(0.59-1.1)$ & 0.10 & $0.79(0.59-1.1)$ & 0.13 & $0.74(0.56-.97)$ & 0.029 & $0.74(0.56-.97)$ & 0.031 \\
\hline $\mathrm{P}_{\text {Trend }} \S$ & 0.19 & - & 0.18 & - & 0.097 & - & 0.089 & - \\
\hline \multicolumn{9}{|l|}{ Neutrophil ${ }^{\uparrow \Lambda}$} \\
\hline Q3 (>5.5-7.1) & $1.2(0.88-1.6)$ & 0.26 & $1.6(1.2-2.2)$ & 0.0029 & $1.2(0.91-1.6)$ & 0.19 & $1.3(0.96-1.7)$ & 0.099 \\
\hline Q4 (>7.1) & $1.6(1.2-2.2)$ & 0.0021 & $1.8(1.3-2.5)$ & 0.0004 & $1.1(0.84-1.5)$ & 0.45 & $1.2(0.87-1.6)$ & 0.31 \\
\hline $\mathrm{P}_{\text {Trend }} \S$ & $<0.0001$ & - & $<0.0001$ & - & 0.30 & - & 0.17 & - \\
\hline \multicolumn{9}{|l|}{$\mathrm{NLR}^{q^{\uparrow \Lambda}}$} \\
\hline Q1 ( $\leq 2.1)$ & 1.0 Referent & - & 1.0 Referent & - & 1.0 Referent & - & 1.0 Referent & - \\
\hline Q2 (>2.1-3.0) & $1.1(0.81-1.5)$ & 0.52 & $1.1(0.78-1.5)$ & 0.67 & $0.85(0.64-1.1)$ & 0.26 & $0.82(0.62-1.1)$ & 0.18 \\
\hline Q3 (>3.0-4.1) & $1.5(1.1-2.0)$ & 0.012 & $1.5(1.1-2.0)$ & 0.015 & $1.2(0.94-1.6)$ & 0.14 & $1.3(0.97-1.7)$ & 0.082 \\
\hline Q4 (>4.1) & $2.0(1.5-2.7)$ & $<0.0001$ & $1.9(1.4-2.6)$ & $<0.0001$ & $1.2(0.94-1.6)$ & 0.12 & $1.3(0.998-1.8)$ & 0.052 \\
\hline
\end{tabular}

¥ , adjusted for age (continuous), BMI (continuous), sex (male, female), race (white, black, East Asian, other), stage (IIIA, IIIB), treatment (cisplatin + etoposide, cisplatin + pemetrexed); ${ }^{\dagger}$, Wald test; " , back-transformed from logarithmic values; ${ }^{\wedge}$, pretreatment (baseline); ${ }^{\S}$, Likelihood ratio test for trend. aHR, adjusted hazard ratio; BMI, body mass index. Cl, confidence interval. ETO, cisplatin + etoposide. HR, unadjusted hazard ratio. NLR, neutrophil-lymphocyte ratio. PEM, cisplatin + pemetrexed. PLR, platelet-lymphocyte ratio. $Q$, quartile.

$(\mathrm{P}=0.0047)$ times were significantly shorter among patients with stage IIIB vs. IIIA disease. There was no significant difference in OS between the two treatment regimens ( $\mathrm{HR}=1.0, \mathrm{P}=0.97)$. The median baseline neutrophil and lymphocyte counts were 5.5 (IQR 2.7) and 1.9 (IQR 0.91), respectively (not shown in Table 1). Patients were followed for a median of 24 months (IQR 22).

\section{Survival outcomes}

No significant association was demonstrated between OS and baseline lymphocyte count, although lymphocyte counts above 2.4 were significantly associated with better PFS than a lymphocyte counts less than or equal to 1.5 ( $\mathrm{HR}=0.74$, $\mathrm{P}=0.029)$. No significant association or linear trend was noted between lymphocyte counts and OS or PFS (Table 2).

Increased baseline neutrophil counts were significantly associated with worse OS, with patients in the $\mathrm{Q}_{4}$ range (>7.1) having a HR of $1.6(\mathrm{P}=0.0021)$ and aHR of 1.8 $(\mathrm{P}=0.0004)$ compared with referents in the $\mathrm{Q}_{1}$ range $(\leq 4.4)$. No significant association was noted for PFS. Increasing baseline neutrophil counts showed a significant trend for worse OS, before and after adjusting for demographic characteristics i.e., age, BMI, cancer type, gender $\left(P_{\text {Trend }}\right.$ $<0.0001)$; however, no such association was noted for PFS.

At baseline, a higher NLR was associated with poorer OS. The five-year OS rate for patients with NLR $\leq 2.1$ was $39 \%$, while for patients with NLR $>4.1$, the rate was $20 \%$, with a significant trend towards decreased OS as NLR increased (Table 3). Higher baseline NLR was associated 
Table 3 Overall and progression-free survival by indicated year following baseline

\begin{tabular}{|c|c|c|c|c|c|c|}
\hline Inflammatory marker & \multicolumn{3}{|c|}{ Overall survival, percentage $(95 \% \mathrm{Cl})$} & \multicolumn{3}{|c|}{ Progression-free survival, percentage $(95 \% \mathrm{Cl})$} \\
\hline \multicolumn{7}{|l|}{$\mathrm{NLR}^{\Uparrow}$} \\
\hline Q1 ( $\leq 2.1)$ & $0.80(0.72-0.85)$ & $0.50(0.41-0.59)$ & $0.39(0.28-0.49)$ & $0.45(0.36-0.54)$ & $0.17(0.11-0.26)$ & $0.13(0.06-0.22)$ \\
\hline Q2 (>2.1-3.0) & $0.82(0.74-0.87)$ & $0.45(0.36-0.53)$ & $0.38(0.29-0.47)$ & $0.53(0.44-0.62)$ & $0.24(0.16-0.32)$ & $0.19(0.12-0.28)$ \\
\hline Q4 (>4.1) & $0.69(0.61-0.76)$ & $0.24(0.16-0.31)$ & $0.20(0.13-0.29)$ & $0.35(0.27-0.44)$ & $0.14(0.08-0.22)$ & $0.12(0.06-0.20)$ \\
\hline
\end{tabular}

", back-transformed from logarithmic values. $\mathrm{Cl}$, confidence interval; $\mathrm{Q}$, quartile.

A

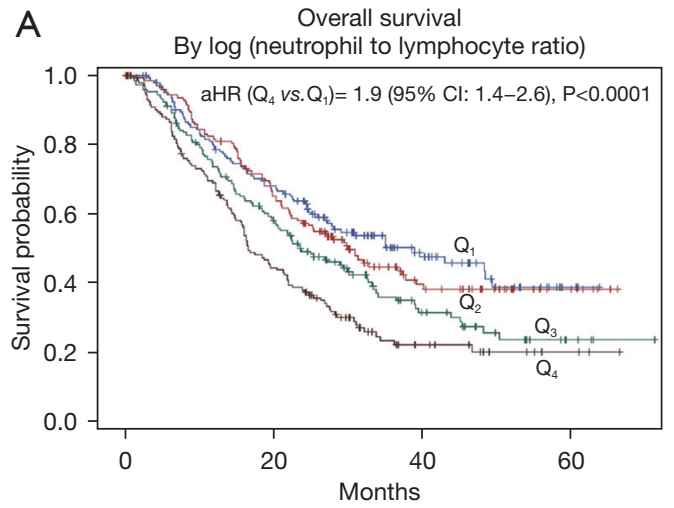

B Progression-free survival

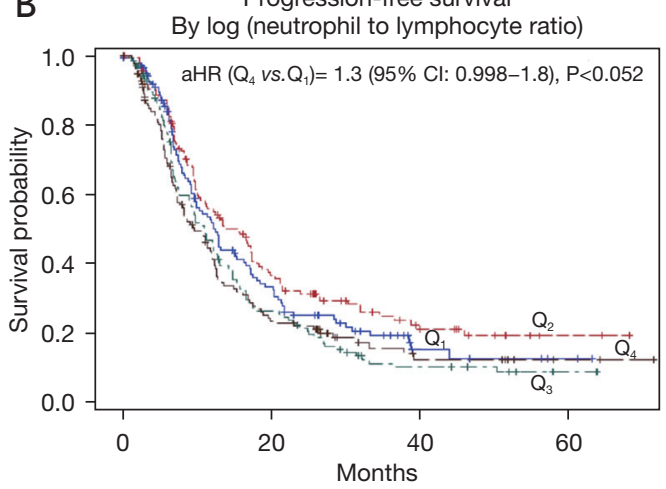

Figure 1 Kaplan-Meier plots for overall survival (A) and progression-free survival (B) by quartile groups $\left(\mathrm{Q}_{1}-\mathrm{Q}_{4}\right)$.

with trends towards shorter PFS. Patients with baseline NLR $\leq 2.1$ had PFS rates of $45 \%$ and $17 \%$ at 1 and 3 years, respectively; conversely, patients with NLR $>4.1$ had PFS of $35 \%$ and $14 \%$ at 1 and 3 years, respectively. Kaplan Meier curves for OS and PFS are shown in Figure 1A,1B.

\section{Discussion}

Systemic inflammation has a pivotal role in the progression of various cancer subtypes including NSCLC by promoting tumor angiogenesis, proliferation and ultimately metastasis. This chronic condition also affects tumor response to cancer treatment, including radiation and chemotherapy. NLR, which is the ratio of the circulating neutrophil and lymphocyte, is an important marker for systemic inflammation. The results of the current study further highlight that this inflammation-based prognostic score is a reliable pretreatment marker for survival outcomes among patients with newly diagnosed stage III NSCLC undergoing chemo-radiation. Our analysis of a large phase III clinical trial dataset supports the findings of several smaller, singlecenter, and/or less-extensive studies, and sets the stage for exploring the prognostic role of NLR in the era of cancer immunotherapy (35).

Our analysis is in alignment with the meta-analysis showing increased baseline NLR is associated with worse survival in NSCLC (36). However, in comparison with the published meta-analysis which has several limitations because of the underlying heterogeneity in the published articles, along with small sample size of individual studies, the current analysis is from a large, randomized dataset supporting the role of baseline NLR values as a significant prognostic marker for locally advanced NSCLC.

Individual lymphocyte and neutrophil counts were associated separately with OS and PFS in our analysis, with the magnitude of the reciprocal effect of lymphocyte counts being considerably smaller than that NLR. While the aHRs for survival were similar for neutrophils counts alone and NLR, we posit that NLR may be a more clinically relevant prognostic marker when applied to individual 
patients. For example, exogenous corticosteroid use causes iatrogenic neutrophilia by directly inhibiting endovascular L-selectin synthesis (37-42). Steroids directly inhibit inflammatory processes, and thus there would not be an associated decrease in circulating lymphocytes typically seen in systemic inflammation $(43,44)$. Correspondingly, NLR in patients treated with corticosteroids would be less pronounced.

As utilization of ICIs become more common in the treatment of NSCLC, the value of systemic inflammation needs to be explored further. This is particularly relevant, given that less than $25 \%$ of patients positively respond to ICIs as monotherapy for NSCLC (45). NLR also may play a role in the selection of patients to enroll in future clinical trials of these agents. There have been conflicting reports in defining the prognostic role of NLR in patients treated with ICIs. A recent systematic review and network meta-analysis of 3,024 patients treated with ICIs reported that high pretreatment NLR is associated with poor PFS and OS (46). In stage III unresectable NSCLC, consolidative durvalumab for one year has become the standard of care based on the PACIFIC trial (47).

Radiation therapy is known to cause lymphopenia more pronounced than chemotherapy alone. For NSCLC, expression of PD-L1 is an acceptable marker to predict response rate to these agents. At present, in the postchemoradiation setting, whether NLR will add to value in predicting outcome is unknown. Further studies are needed to investigate the role of the immune environment and high levels of NLR caused by chemo-radiation in predicting response to consolidative durvalumab.

To the best of our knowledge, the current study is the first large-scale analysis examining the prognostic survival potential of pretreatment NLR in patients diagnosed with locally advanced, stage IIIA/B NSCLC utilizing a prospective clinical trial dataset. Additionally, only nonsquamous cell cancers were included, eliminating variability between different histologies.

Biomarker studies often yield inconsistent conclusions owing to poor study design, methodological problems, nonstandardized assays, misleading statistical analyses, and a general lack of rigor and reproducibility (6). Finding reliable and valid prognostic markers that predict clinical outcomes and aid in the selection of a targeted treatment plan remains paramount to the field oncology. Previously published reports of NLR as a prognostic marker for NSCLC either had a small sample size (48-50) or were retrospective in nature $(51,52)$, included patients with non-stage III disease (27,53-57), or surgical series and/or analyzed outcomes other than mortality. In contrast, our study analyzed data obtained from a phase III clinical trial of stage IIIA/B NSCLC patients and exclusively considered pretreatment NLR and survival outcomes. Additionally, we avoided arbitrary and data-dependent cutoff points by defining comparison groups based on their exact quartile values.

Certain limitations should be noted when considering our results. The NLR was not the a priori aim of the initial trial, which also preceded the usage of ICIs that are now critical tools for achieving favorable response in NSCLC. Clinical trials also may not be reflective of the general population, owing to restrictive inclusion and exclusion criteria (41). Information on competing causes of deaths was not available in this analysis. Consequently, all causes of death were independently censored, which may have inflated the proportion of deaths attributable to lung cancer in our Cox-proportional models (58). Nonetheless, the effect of competing risk in advanced stage lung cancer is nominal in comparison with other less invasive tumors.

Overall, pre-treatment NLR ratio is an important prognostic marker for patients presenting with locally advanced non-squamous NSCLC who underwent definitive chemoradiation. Future large-scale studies are needed to investigate the value of post-chemoradiation and pre-durvalumab NLR, or other systemic immune-inflammation indexes, in stage III NSCLC. Targeted therapies directed at driver gene mutations (e.g., anti-EGFR, anti-VEGF, anti-ALK, MET, and BRAF) also are promising therapeutic agents in the context of NLR and NSCLC survival outcomes. Carefully examining the effect modification of NLR status and the use of targeted therapies such as Afatinib, Alectinib, Cabozantinib, Ceritinib, E Crizotinib, Dabrafenib/Trametinib, Gefitinib, Osimertinib, Rlotinib, merits further consideration (59).

\section{Conclusions}

NSCLC is a complex disease, and few validated markers are currently available for predicting mortality outcomes of this cancer. In contrast to exploratory retrospective studies, our hypothesis-driven analysis provides confirmatory evidence that higher pre-treatment NLR is a consistent indicator of decreased OS and PFS in stage IIIA/B NSCLC. Future research will benefit from exploring the increased prognostic value of NLR in the setting of various genetic mutations and molecular markers. 


\section{Acknowledgments}

Data used in these analyses are from Lilly trial H3E-MCJMIG (clinicaltrial.gov ID: NCT00686959) and were accessed using www.clinicalstudydatarequest.com.

Funding: None.

\section{Footnote}

Reporting Checklist: The authors have completed the REMARK reporting checklist. Available at https://dx.doi. org/10.21037/jtd-21-1018

Data Sharing Statement: Available at https://dx.doi. org/10.21037/jtd-21-1018

Conflicts of Interest: All authors have completed the ICMJE uniform disclosure form (available at https:// dx.doi.org/10.21037/jtd-21-1018). T.B.: Demos Medical, AstraZeneca, Galera Therapeutics, Electa. D.B.: Seattle Genetics, Abbvie, Rafael Pharmaceuticals. M.M.: AstraZeneca, ViewRay, Varian. The other authors have no conflicts of interest to declare.

Ethical Statement: The authors are accountable for all aspects of the work in ensuring that questions related to the accuracy or integrity of any part of the work are appropriately investigated and resolved. The study was conducted in accordance with the Declaration of Helsinki (as revised in 2013). This was an analysis of de-identified data from the PROCLAIM study (Eli Lilly). No patients were involved or contacted for the current manuscript and the analysis was considered IRB exempt by Code of Federal Regulations 45 part 46.101(b).

Open Access Statement: This is an Open Access article distributed in accordance with the Creative Commons Attribution-NonCommercial-NoDerivs 4.0 International License (CC BY-NC-ND 4.0), which permits the noncommercial replication and distribution of the article with the strict proviso that no changes or edits are made and the original work is properly cited (including links to both the formal publication through the relevant DOI and the license). See: https://creativecommons.org/licenses/by-nc-nd/4.0/.

\section{References}

1. Division of Cancer Prevention and Control. Centers for
Disease Control and Prevention, 2021. Available online: https://www.cdc.gov/cancer/lung/

2. Molina JR, Yang P, Cassivi SD, et al. Non-small cell lung cancer: epidemiology, risk factors, treatment, and survivorship. Mayo Clin Proc 2008;83:584-94.

3. Chen Z, Fillmore CM, Hammerman PS, et al. Non-smallcell lung cancers: a heterogeneous set of diseases. Nat Rev Cancer 2014;14:535-46.

4. Botticella A, Mezquita L, Le Pechoux C, et al. Durvalumab for stage III non-small-cell lung cancer patients: clinical evidence and real-world experience. Ther Adv Respir Dis 2019;13:1753466619885530.

5. National Cancer Institute. Lung Cancer 2021. Available online: https://www.cancer.gov/types/lung

6. McShane LM, Altman DG, Sauerbrei W, et al. REporting recommendations for tumour MARKer prognostic studies (REMARK). Br J Cancer 2005;93:387-91.

7. Balkwill F, Mantovani A. Inflammation and cancer: back to Virchow? Lancet 2001;357:539-45.

8. Keit E, Coutu B, Zhen W, et al. Systemic inflammation is associated with inferior disease control and survival in stage III non-small cell lung cancer. Ann Transl Med 2021;9:227.

9. Sparmann A, Bar-Sagi D. Ras-induced interleukin-8 expression plays a critical role in tumor growth and angiogenesis. Cancer Cell 2004;6:447-58.

10. Balkwill F, Charles KA, Mantovani A. Smoldering and polarized inflammation in the initiation and promotion of malignant disease. Cancer Cell 2005;7:211-7.

11. Staller P, Sulitkova J, Lisztwan J, et al. Chemokine receptor CXCR4 downregulated by von Hippel-Lindau tumour suppressor pVHL. Nature 2003;425:307-11.

12. Lee Y, Kim SH, Han JY, et al. Early neutrophil-tolymphocyte ratio reduction as a surrogate marker of prognosis in never smokers with advanced lung adenocarcinoma receiving gefitinib or standard chemotherapy as first-line therapy. J Cancer Res Clin Oncol 2012;138:2009-16.

13. De Larco JE, Wuertz BR, Furcht LT. The potential role of neutrophils in promoting the metastatic phenotype of tumors releasing interleukin-8. Clin Cancer Res 2004;10:4895-900.

14. Hart IR, Fidler IJ. Role of organ selectivity in the determination of metastatic patterns of B16 melanoma. Cancer Res 1980;40:2281-7.

15. Kobayashi N, Usui S, Kikuchi S, et al. Preoperative lymphocyte count is an independent prognostic factor in node-negative non-small cell lung cancer. Lung Cancer 2012;75:223-7. 
16. Stanley KE. Prognostic factors for survival in patients with inoperable lung cancer. J Natl Cancer Inst 1980;65:25-32.

17. Zhao Q, Chen G, Ye L, et al. Treatment-duration is related to changes in peripheral lymphocyte counts during definitive radiotherapy for unresectable stage III NSCLC. Radiat Oncol 2019;14:86.

18. Zhang J, Huang SH, Li H, et al. Preoperative lymphocyte count is a favorable prognostic factor of disease-free survival in non-small-cell lung cancer. Med Oncol 2013;30:352.

19. Bremnes RM, Al-Shibli K, Donnem T, et al. The role of tumor-infiltrating immune cells and chronic inflammation at the tumor site on cancer development, progression, and prognosis: emphasis on non-small cell lung cancer. J Thorac Oncol 2011;6:824-33.

20. Gaissmaier L, Christopoulos P. Immune Modulation in Lung Cancer: Current Concepts and Future Strategies. Respiration 2020. [Epub ahead of print]. doi: 10.1159/000510385.

21. Lee F, Yang PS, Chien MN, et al. An Increased Neutrophil-to-Lymphocyte Ratio Predicts Incomplete Response to Therapy in Differentiated Thyroid Cancer. Int J Med Sci 2018;15:1757-63.

22. Kim YJ, Lee I, Chung YS, et al. Pretreatment neutrophil-to-lymphocyte ratio and its dynamic change during neoadjuvant chemotherapy as poor prognostic factors in advanced ovarian cancer. Obstet Gynecol Sci 2018;61:227-34.

23. Lin AJ, Rao YJ, Chin RI, et al. Post-operative radiation effects on lymphopenia, neutrophil to lymphocyte ratio, and clinical outcomes in palatine tonsil cancers. Oral Oncol 2018;86:1-7.

24. Zhou XL, Li YQ, Zhu WG, et al. Neutrophil-to-lymphocyte ratio as a prognostic biomarker for patients with locally advanced esophageal squamous cell carcinoma treated with definitive chemoradiotherapy. Sci Rep 2017;7:42581.

25. Liu D, Huang Y, Li L, et al. High neutrophil-tolymphocyte ratios confer poor prognoses in patients with small cell lung cancer. BMC Cancer 2017;17:882.

26. Jang WS, Cho KS, Kim KH, et al. Prognostic impact of preoperative neutrophil-to-lymphocyte ratio after radical prostatectomy in localized prostate cancer. Prostate Cancer Prostatic Dis 2016;19:298-304.

27. Cannon NA, Meyer J, Iyengar P, et al. Neutrophillymphocyte and platelet-lymphocyte ratios as prognostic factors after stereotactic radiation therapy for early-stage non-small-cell lung cancer. J Thorac Oncol 2015;10:280-5.

28. Diem S, Schmid S, Krapf M, et al. Neutrophil-to-
Lymphocyte ratio (NLR) and Platelet-to-Lymphocyte ratio (PLR) as prognostic markers in patients with nonsmall cell lung cancer (NSCLC) treated with nivolumab. Lung Cancer 2017;111:176-81.

29. Cupp MA, Cariolou M, Tzoulaki I, et al. Neutrophil to lymphocyte ratio and cancer prognosis: an umbrella review of systematic reviews and meta-analyses of observational studies. BMC Med 2020;18:360.

30. Senan S, Brade A, Wang LH, et al. PROCLAIM: Randomized Phase III Trial of Pemetrexed-Cisplatin or Etoposide-Cisplatin Plus Thoracic Radiation Therapy Followed by Consolidation Chemotherapy in Locally Advanced Nonsquamous Non-Small-Cell Lung Cancer. J Clin Oncol 2016;34:953-62.

31. Therasse P, Arbuck SG, Eisenhauer EA, et al. New guidelines to evaluate the response to treatment in solid tumors. European Organization for Research and Treatment of Cancer, National Cancer Institute of the United States, National Cancer Institute of Canada. J Natl Cancer Inst 2000;92:205-16.

32. International Council for Harmonisation of Technical Requirements for Pharmaceuticals for Human Use (ICH). Available online: www.ich.org

33. Grambsch PM, Therneau TM. Proportional hazards tests and diagnostics based on weighted residuals. Biometrika 1994;81:515-26.

34. Efird JT. Goldilocks Rounding: Achieving Balance Between Accuracy and Parsimony in the Reporting of Relative Effect Estimates. Cancer Inform 2021;20:1176935120985132.

35. Russo A, Russano M, Franchina T, et al. Neutrophil-toLymphocyte Ratio (NLR), Platelet-to-Lymphocyte Ratio (PLR), and Outcomes with Nivolumab in Pretreated NonSmall Cell Lung Cancer (NSCLC): A Large Retrospective Multicenter Study. Adv Ther 2020;37:1145-55.

36. Yin Y, Wang J, Wang X, et al. Prognostic value of the neutrophil to lymphocyte ratio in lung cancer: A metaanalysis. Clinics (Sao Paulo) 2015;70:524-30.

37. Filep JG, Delalandre A, Payette Y, et al. Glucocorticoid receptor regulates expression of L-selectin and CD11/ CD18 on human neutrophils. Circulation 1997;96:295-301.

38. Burton JL, Kehrli ME Jr, Kapil S, et al. Regulation of L-selectin and CD18 on bovine neutrophils by glucocorticoids: effects of cortisol and dexamethasone. J Leukoc Biol 1995;57:317-25.

39. Weber PS, Toelboell T, Chang LC, et al. Mechanisms of glucocorticoid-induced down-regulation of neutrophil L-selectin in cattle: evidence for effects at the gene- 
expression level and primarily on blood neutrophils. J Leukoc Biol 2004;75:815-27.

40. Smith CW. Endothelial adhesion molecules and their role in inflammation. Can J Physiol Pharmacol 1993;71:76-87.

41. Van Eeden S, Miyagashima R, Haley L, et al.

L-selectin expression increases on peripheral blood polymorphonuclear leukocytes during active marrow release. Am J Respir Crit Care Med 1995;151:500-7.

42. Török C, Lundahl J, Hed J, et al. Diversity in regulation of adhesion molecules (Mac-1 and L-selectin) in monocytes and neutrophils from neonates and adults. Arch Dis Child 1993;68:561-5.

43. Cronstein BN, Kimmel SC, Levin RI, et al. A mechanism for the antiinflammatory effects of corticosteroids: the glucocorticoid receptor regulates leukocyte adhesion to endothelial cells and expression of endothelial-leukocyte adhesion molecule 1 and intercellular adhesion molecule 1. Proc Natl Acad Sci U S A 1992;89:9991-5.

44. Goulding NJ, Euzger HS, Butt SK, et al. Novel pathways for glucocorticoid effects on neutrophils in chronic inflammation. Inflamm Res 1998;47 Suppl 3:S158-65.

45. Onoi K, Chihara Y, Uchino J, et al. Immune Checkpoint Inhibitors for Lung Cancer Treatment: A Review. J Clin Med 2020;9:1362.

46. Tan PS, Aguiar P Jr, Haaland B, et al. Comparative effectiveness of immune-checkpoint inhibitors for previously treated advanced non-small cell lung cancer A systematic review and network meta-analysis of 3024 participants. Lung Cancer 2018;115:84-8.

47. Antonia SJ, Villegas A, Daniel D, et al. Durvalumab after Chemoradiotherapy in Stage III Non-Small-Cell Lung Cancer. N Engl J Med 2017;377:1919-29.

48. Kang KH, Efird JT, Sharma N, et al. Prognostic potential of neutrophil-to-lymphocyte ratio and lymphocyte nadir in stage III non-small-cell lung cancer. Future Oncol 2017;13:1405-14.

49. Palomar-Abril V, Soria-Comes T, Campos ST, et al. Dynamic evaluation of neutrophil-to-lymphocyte ratio as prognostic factor in stage III non-small cell lung cancer treated with chemoradiotherapy. Clin Transl Oncol 2020;22:2333-40.

50. Yucel S, Bilgin B. The prognostic values of systemic immune-inflammation index and derived neutrophillymphocyte ratio in EGFR-mutant advanced non-small cell lung cancer. J Oncol Pharm Pract 2021;27:71-7.
51. Guo D, Jin F, Jing W, et al. Incorporation of the SUVmax Measured From FDG PET and Neutrophilto-lymphocyte Ratio Improves Prediction of Clinical Outcomes in Patients With Locally Advanced Non-smallcell Lung Cancer. Clin Lung Cancer 2019;20:412-9.

52. Tomita M, Ayabe T, Maeda R, et al. Comparison of Inflammation-Based Prognostic Scores in Patients undergoing Curative Resection for Non-small Cell Lung Cancer. World J Oncol 2018;9:85-90.

53. Gao Y, Zhang H, Li Y, et al. Preoperative increased systemic immune-inflammation index predicts poor prognosis in patients with operable non-small cell lung cancer. Clin Chim Acta 2018;484:272-7.

54. Shoji F, Kozuma Y, Toyokawa G, et al. Complete Blood Cell Count-Derived Inflammatory Biomarkers in EarlyStage Non-Small-Cell Lung Cancer. Ann Thorac Cardiovasc Surg 2020;26:248-55.

55. Derman BA, Macklis JN, Azeem MS, et al. Relationships between longitudinal neutrophil to lymphocyte ratios, body weight changes, and overall survival in patients with non-small cell lung cancer. BMC Cancer 2017;17:141.

56. Berardi R, Santoni M, Rinaldi S, et al. Pre-treatment systemic immune-inflammation represents a prognostic factor in patients with advanced non-small cell lung cancer. Ann Transl Med 2019;7:572.

57. Zer A, Sung MR, Walia P, et al. Correlation of Neutrophil to Lymphocyte Ratio and Absolute Neutrophil Count With Outcomes With PD-1 Axis Inhibitors in Patients With Advanced Non-Small-Cell Lung Cancer. Clin Lung Cancer 2018;19:426-434.e1.

58. Gooley TA, Leisenring W, Crowley J, et al. Estimation of failure probabilities in the presence of competing risks: new representations of old estimators. Stat Med 1999;18:695-706.

59. Zhu QG, Zhang SM, Ding XX, et al. Driver genes in nonsmall cell lung cancer: Characteristics, detection methods, and targeted therapies. Oncotarget 2017;8:57680-92.

Cite this article as: Biswas T, Gawdi R, Jindal C, Iyer S, Kang KH, Bajor D, Machtay M, Choi YM, Efird JT. Pretreatment neutrophil-to-lymphocyte ratio as an important prognostic marker in stage III locally advanced non-small cell lung cancer: confirmatory results from the PROCLAIM phase III clinical trial. J Thorac Dis 2021;13(10):5617-5626. doi: 10.21037/jtd-21-1018 